\title{
Inhaltsverzeichniss des vier und sechzigsten Bandes.
}

De superficiebus in planum explicabilibus primorum septem ordinum. Auctore

H. Schwarz. . . . . . . . . . . . . . . . . . . . . . Seite 1

Ueber die Transformation der Abelschen Functionen erster Ordnung. Von

Herrn Königsberger zu Greifswald. . . . . . . . . . . . . . - 17

Ueber diejenigen ebenen Curven, deren Coordinaten rationale Functionen

eines Parameters sind. Von Herrn A. Clebsch zu Giessen. . . . . . - 43

Ueber die Flăchen vierten Grades, auf welchen Schaaren von Kegelschnitten liegen. Von Herrn Kummer. (Abgedruckt aus dem Monatsbericht der

Berliner Akademie rom 16. Juli 1863.) . . . . . . . . . . . . - 66

Note zur vorstebenden Abhandlung. Von Herrn Weierstrass. (Abgedruckt

aus dem Monatsbericht der Berliner Akademio vom 16. Juli 1863.) . . - 77

Ueber die Steinersche Fläche vierten Grades. Von Herrn Schröter zu Breslau.

(Abgedruckt aus dem Monatsbericht der Berliner Akademie rom

26. Nov. 1863.) . . . . . . . . . . . . . . . . . . . . . 779

Ueber dfe. Elimination aus zwei Gleichungen dritten Grades. Von Herrn

A. Clebsch zn Giessen. . . . . . . . . . . . . . . . . . . - 95

Ueber die Singularităten algebraischer Curven. Von Demselben. . . . . - 98

Sur l'hypocycloïde à trois rebroussements. Par M. L. Cremona à Bologne. - 101

Note zur vorstehenden Abhandlung. Von Herrn A. Clebsch zu Giessen. . . - 124

Ueber das einem Kegelschnitte umschriebene und einem andern einbeschriebene

Polygon. Von den Herren J. Rosanes und M. Pasch zu Breslau. . . - 126

Suite des recherches sur l'élimination et la théorie des courbes. Par M.

A. Cayley à Cambridge. . . . . . . . . . . . . . . . . . -167

Note sur la surface du quatrième ordre de Steiner. Par M. A. Cayley à

Cambridge. . . . . . . . . . . . . . . . . . . . . . -172

Ueber eine neue analytische Behandlungsweise der Brennpunkte. Von

Herrn Siebeck zu Liegnitz. . . . . . . . . . . . . . . . . - 175

Note uber die Evoluten sphärischer Curven. Von Herrn Mehler zu Danzig. — 183 
Ueber Curvenbttschel, die sich gegenseitig bertlhren. Von Herrn J. N. Bischoff zu Zweibrttcken. . . . . . . . . . . . . . . . . . . . . Seite 185

Bemerkung zu „Hesse, Zerlegung der Bedingung für die Gleichheit der Hauptaxen eines auf einer Oberflăche zweiter Ordnung liegenden Kegelschnittes in die Summe von Quadraten." (Bd. 60, p. 305.) Von Herrp Henrici zu Kiel. . . . . . . . . . . . . . . . . . . . . . . . -187

Ueber die Bestimmung der Gestalt einer krummen Oberfläche durch lokale Messungen auf derselben. Von Herm E. B. Christoffel in Zurich. . . - 193

Ueber diejenigen Curven, deren Coordinaten sich als elliptische Functionen eines Parameters darstellen lassen. Von Herrn A. Clebsch zu Giessen. - 210

Hauptlehrsåtze der Theorie der partiellen Differentialgleichungen erster Ordnung mit drei Variabeln. Von Herrn Paul du Bois-Reymond. . . . . -271

Ueber eine Vorsichtsmassregel beim Gebrauch des Princips der virtuellen Geschwindigkeiten. Von Herrn Bertram. . . . . . . . . . . . - 284

Ueber einige von Steiner behandelte Curven. Von Herrn A. Clebsch zu Giessen. - 288

Extrait d'une lettre de M. Hermite à M. Borchardt. . . . . . . . . . - 294

De investigando ordine systematis aequationnm differentialium vulgarium cujuscunque. Ex ill. C. G. J. Jacobi manuscriptis posthumis in medium protulit C. W. Borchardt. . . . . . . . . . . . . . . . . . -297

Zur Theorie der einwerthigen Potentiale. Von Herrn E. B. Christoffel in Zurich. . . . . . . . . . . . . . . . . . . . . . . . -321

Note sur les singularités supérieures des courbes planes. Par M. A. Cayley à Cambridge. . . . . . . . . . . . . . . . . . . . . . -369

Ueber die Anzahl der willkurlichen Constanten in algebraischen Functionen.

Von Herrn G. Roch in Halle. . . . . . . . . . . . . . . . -372 